\title{
Evaluation and Analysis of Bio-Inspired Optimization Techniques for Bill Estimation in Fog Computing
}

\author{
Hafsa Arshad ${ }^{1}$, Hasan Ali Khattak ${ }^{1 *}$, Munam Ali Shah ${ }^{1}$, Assad Abbas ${ }^{1}$ and Zoobia Ameer ${ }^{2}$ \\ ${ }^{1}$ Department of Computer Science, COMSATS University Islamabad, Islamabad 44500, Pakistan \\ ${ }^{2}$ Department of Physics, Shaheed Benazir Bhutto Women University, Peshawar 25500, Pakistan
}

\begin{abstract}
In light of constant developments in the realm of Information Communication and Technologies, large-scale businesses and Internet service providers have realized the limitation of data storage capacity available to them. This led organizations to cloud computing, a concept of sharing of resources among different service providers by renting these resources through service level agreements. Fog computing is an extension to cloud computing architecture in which resources are brought closer to the consumers. Fog computing, being a distinct from cloud computing as it provides storage services along with computing resources. To use these services, the organizations have to pay according to their usage. In this paper, two nature-inspired algorithms, i.e. Pigeon Inspired Optimization (PIO) and Binary Bat Algorithm (BBA) are compared to regulate the effective management of resources so that the cost of resources can be curtailed and billing can be achieved by calculating utilized resources under the service level agreement. PIO and BBA are used to evaluate energy utilization by cloudlets or edge nodes that can be used subsequently for approximating the utilization and bill through a Time of Use pricing scheme. We appraise abovementioned techniques to evaluate their performance concerning the bill estimation based on the usage of fog servers. With respect to the utilization of resources and reduction in the bill, simulation results have revealed that the BBA gives pointedly better results than PIO.
\end{abstract}

Keywords-Cloud computing; fog computing; bio-inspired algorithms; pricing; cloudlets

\section{INTRODUCTION}

In order to enhance the efficiency and performance in distributed computing, components of a software system are distributed or shared among multiple systems. Cloud computing is regarded as a type of distributed computing that comprises the services available to users from distant locations. It is an evolving computing architecture that counts on shared computing resources to handle applications in spite of having local servers. The users can utilize through cloud computing, several services and resources such as processing and storage through internet. The on-demand delivery of the Information Technology (IT) resources is guaranteed by charging the services through the pay as you go model. The cloud customers pay to the service providers for providing the services directly to the end users. One can acquire as many resources as required by remitting the resources used. In fact, the cloud computing has either fully removed or has substantially reduced the costs of developing and maintaining the IT infrastructure for small and medium sized enterprises (SME's). Due to some intrinsic issues, many applications cannot work effectively in the cloud environment. For example, because of the low bandwidth the data transmission to the cloud can not be at the same rate at which it is generated. Therefore, noteworthy delays can be faced which are unacceptable in certain cases. Fog computing has established its efficacy to overcome several issues of distributed computing, including inefficient resource management, Quality-of-Service (QoS), security, and privacy issues. The data in the fog computing environment is processed locally in a virtual platform at a much faster pace as compared to a centralized cloud server.

The term Fog computing was introduced by Cisco Systems for the first time, which is also known as edge computing. It facilitates in wireless data transfer in the Internet of Things (IoT) model by taking the computing power near to the Edge of the network so that the devices have an easy and vigorous access. The key idea of fog computing is to boost the efficiency and minimize the data transmissions in predominance to cloud for analysis, storage, and processing purposes. One of the key benefits is the lesser dormancy for devices and lesser network load on the internet mainstay. There are numerous application domains of fog computing, including softwaredefined networks, vehicular networks, smart grid, smart cities and smart buildings [1]. The long-term analytic processing is carried out in the cloud environment whereas short term data analysis takes place at the fog servers or edge nodes. It is also worth mentioning that the fog computing cannot be completely replaced by the cloud. It only strengthens the cloud computing by decreasing the data sent to the cloud for processing through the mainstay Internet.

As stated above, the fog computing lessens the bulk of data sent to the cloud, subsequently conserving the network bandwidth. Furthermore, it also advances the system response time by keeping the data close to the edge of the network and making the data available promptly. Moreover, the fog computing supports agility and minimizes the dormancy or latency.

The organizations rent the services from the fog service providers. However, it is imperative to efficiently manage and utilize the available resources to reduce the bills. One way to reduce the costs is to optimally utilize the resources through the Time of Use (ToU) pricing model. To illustrate the scenario, an example of smart homes is considered where smart meters are placed for measuring the energy consumed that is subsequently used for bill calculation [2], [3]. Fig. 1 shows general three layered architecture of edge nodes or cloudlets. In the top- 


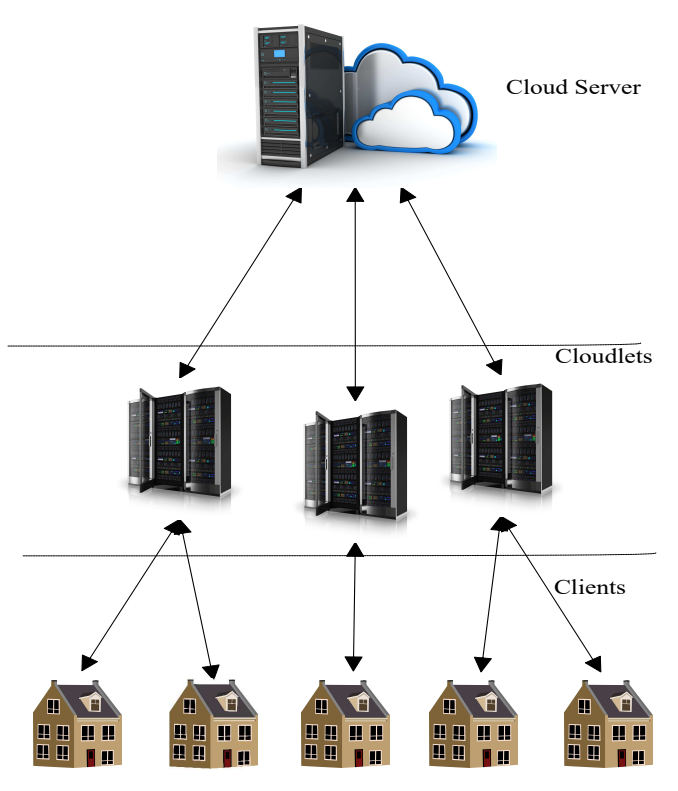

Fig. 1. General 3-layered architecture.

most layer, cloud server is placed. The second (middle) layer is the application layer that comprises of cloudlets while, the bottom layer which in fact is the third layer contains the clients or consumers of the resources. The consumers or clients can manage the cost and energy consumed, by optimal scheduling. With the increasing demand of cloud services, the necessity to estimate the cost of the consumed resources has increased to large extend. Here, our objective is to estimate the bills on the basis of the energy consumed by the cloudlets by using the bio-inspired techniques, i.e., Pigeon Inspired Optimization (PIO) and the Binary Bat Algorithm (BBA). As a result, the resource utilization can be adjusted in response to the pricing scheme.

In this paper, we employ two bio-inspired optimization techniques, namely, PIO and BBA, to determine the optimal solution for calculating the bills based on the usage of the cloudlets. Along with bio-inspired techniques, ToU pricing signal is used that actually favours the efficient utilization of the system along with the reduction in the overall costs that will eventually benefit both the customers as well as service providers. Through ToU rating scheme, one can get to know when and how much energy is being consumed and likewise consumers also get benefit as they can save their operational costs. The echolocative behaviour of bats is used in the BBA [4]-[6] while homing behaviour of pigeons is considered in the PIO [7]-[9] to obtain the optimal solution. The reason of selection of these two techniques is that they perform better than many other techniques. BBA performed better than PSO and GA [4], [5] and PIO outpaced EDE in [7], [8]. These optimization algorithms perform better than other algorithms in terms of approximating the cost and resource which are being utilized.

The paper below has been presented as follows. Section II presents the related work and the proposed model has been explained in Section III. In Section IV, results and simulations of the proposed system have been explained and the Section $\mathrm{V}$ settles the paper and highpoints the future work.

\section{RELATED WORK}

Being a nascent computing technology, fog computing and cloudlet resource management are under consideration by many scholars. The literature review done in this paper is purely based on cost estimation techniques which are utilized for determining the bills based on the resource usage of cloudlet. State-of-the-art work is given in Table I, stating the contributions of the researchers in this regard. Cost, resource management, scheduling and latency are the key features which are considered along with several parameters including servers, energy consumption, time, memory and throughput.

The authors in [10], proposed a cost makespan aware scheduling heuristic which is a cost scheduling algorithm. The objective of this work is to acquire the balance between the cost for the utilization of cloud resources and the performance of application execution. However, this proposed scheduling can be extended for large scale applications by keeping energy efficiency in mind. In [11], the authors proposed greedy heuristics for Basic Service Placement Problem (BSPP) and Cost-aware Service Placement Problem (CSPP). The main aim of this work is to help service providers to minimize the access latency and reduce the cost of service providers, including resource usages and service placements updating cost on cloudlets. However, privacy and security of the users mobility is ignored. The authors in [12], have proposed a Cost Deadline Based (CDB) task scheduling algorithm in order to schedule tasks with cloudsim. The main goal of this work is to reduce the rate of missed deadline and help to dene cost in the perspective of both user and provider. Efficient utilization of resources is not considered in this work.

The authors in [13], have proposed a heuristic based workflow scheduling scheme using cloud-pricing model. Scheduling approaches include two phases; VM packing and Multi Request to Single Resource (MRSR) scheme. The proposed work reduces the number of entailed VM instances and obtain a cost saving while guaranteeing the users SLA. The cost is reduced by $30 \%$ by using this scheme as compared to other conventional work ow scheduling schemes. However, processing and communication cost of tasks are not considered. In [14], priority-based load balancing approach is proposed that schedules and migrates the VM corresponding to the weights assigned to each virtual machine. The goal of this work is to provide enhanced services to the users who are giving more revenue to the service provider. The drawback of this work is that xed threshold values are used. Cost saving super professional executor (Suprex) with auto-scaling mechanism is proposed by Aslanpour et al. in [15]. The aim of this work is to provide an executor with the capability to isolate the surplus VM till the billed hour is terminated in order to overcome the challenge of postponed VM startup (Suprex). Suprex executor, reduces the renting cost of VM by $7 \%$. However, in some situations, this executor results in lower utilization. In [16], a framework for enhanced resource allocation and prediction on the basis of traits and characteristics of a customer is proposed by a cloud broker in the cloud federation environment. On the basis of past record or data of each customer, the resources and prices are determined. However, QoS is ignored in this work. The authors in [17], have proposed a Science Gateway Cloud (SGC)platform and a cost adaptive resource management scheme along with work ow scheduling scheme 
with a division policy. Although, deadline assurance and cost reduction can be gratified simultaneously, however, cost performance degradation occurs when resource pool management is inefficiently done.

The aim of [18], is to lessen the cost for each queue and the sum cost for both mobile user and the CSP. Algorithms for energy cost minimization, while verifying finite processing delay are proposed in this work. Since, single CSP is used in this work hence, quality of offloading service is sacrificed which can further be enhanced by using multiple CSPs. The authors in [19], have proposed dual side dynamic control algorithms for cost-delay trade-offs of mobile users and CSP in MCC for which cooperation and non-cooperation scenarios are considered.In non-cooperation scenario,the users and the CSP are minimizing their own cost for prescribed delay constraints while in cooperation scenario they are minimizing the social cost for stated processing delay constraints. However, QoS and deadlines ignored in this work.

Cheng et al. in [20], have proposed a deep reinforcement learning (DRL) based system with resource provisioning and task scheduling to minimize the energy cost for CSPs with huge amount of user requests and large-scale data centers. ToU and RTP are the pricing signals applied in this work along with Pay-As-You-Go billing contract or agreement. Energy cost efficiency is improved $320 \%$ and $144 \%$ runtime reduction is achieved. However, dependencies are involved while dealing with large amount of user requests. In [21], the authors have proposed a mathematical framework for dynamic on demand pricing model using IaaS cloud service instances by considering users and providers utility. Genetic algorithm is used for optimized estimation and minimized execution cost. Comparative scrutiny of the intended framework with a utility based pricing model in terms of minimum bill calculation is performed in this work. However, dynamic behaviour of network is not analyzed. The authors in [22], have proposed a load balancing with optimal cost scheduling algorithm to minimize cost and processing power. Already accommodated requests are rescheduled to make space for new upcoming requests for cost optimization at CSP. This algorithm does not operate when all the VMs are busy in the data centers and the upcoming new requests are in waiting condition.

In [23], a negotiation-based iterative approach for task scheduling (NBTS) is proposed to minimize the bill under dynamic energy pricing. The proposed algorithm ends when total energy cost is not diminished. Up to $51.8 \%$ improvement is achieved in electric bill reduction. The paper [24] is the extension of [23] as they both are addressing the same problem. The authors in [24], have proposed a negotiation-based cost minimization (NBCM) algorithm in order to minimize the energy cost of users. Along with that task scheduling (NBTS) and energy storage control (NBSC) systems are also proposed. The main aim of this work is to schedule electricity consumption in a way that the electric bill of the users can be minimized. The total energy cost reduction of $64.22 \%$ is achieved as compared to the baseline methods. Both [23] and [24] have used dynamic energy pricing including ToU and total power consumptiondependent.

The authors in [25], have proposed a model to optimize the resource utilization and cost reduction using dynamic VM provisioning in the cloud. A cost reduction of $16 \%$ is achieved using this model. However, pricing condition applied to spot instances can be further improved. In [26], an admission cost model is proposed for modelling different resource consumptions. The goal of this work is to enhance the system throughput in a cloudlet environment. A storage extension for the existing cloudsim framework to enable simulations of storage as-a-Service (STaaS) components are proposed by Sturm et al. in [27]. For validation of the extension, resource utilization and the cost that arises due to the usage of STaaS clouds are evaluated. However, there is no mechanism for dealing with complex SLAs and realistic pricing models are not used. The authors in [28], have proposed a VM placement scheme to resolve the cost optimization problem along with that VM reallocation grounded on resource utilization-aware activities is also proposed. The objective of this work is to lower the operating cost so that the performance degradation is lessened than the threshold. However, it does not reflect overall trends i.e., temporary resource utilization is done. In [29], Cost oriented model (CoM) is proposed to optimally allocate cloud computing resources for demand side management. The aim of this model is to reduce the rental cost of cloud resources. Amazon cloud computing service-based pricing scheme along with ToU and RTP for demand side management is used in this paper. QoS metric-based resource provisioning technique is proposed in [30] for the cloud computing environment, in order to minimize the cost of cloud workloads and execution time along with other QoS parameters. However, resource utilization can be further improved. The authors in [31], have proposed an efficient online algorithm for dynamic joint VM pricing, scheduling of job and server provisioning containing geo-distributed data centers in order to maximize profit of a cloud provider. Though the backup resources are expensive or job drop penalty is high.

The algorithm proposed in [32], balances the load effectively among VMs by mapping tasks on the basis of foraging behaviour of honey bees. It also minimizes the cost of consuming virtual machine instances. Although, the load of independent tasks is considered for balancing, yet the load of dependent tasks is not considered in this work. In [33], a provably-efficient online dynamic scheduling and pricing (Dyn-SP) algorithm for delay tolerant batch services is proposed to amplify the profit of service provider. It produces close-to-optimal profit. This algorithm is limited to 2 service classes although multiple service classes can be used. The authors in [34], have proposed bi-level cost-wise optimization approach to schedule the consumption level of customers to attain the optimal performance regarding energy minimization cost. However, security is ignored. In [35], the authors have proposed cost optimization algorithm, determining that which resources should be taken on lease from public cloud to accomplish the work ow execution within deadline. Level based scheduling algorithm also known as sub-deadline of work ow is also proposed. Cost based resource allocation strategy is proposed in [36], that will optimize the providers profit along with load balancing technique. The objective of this work is to reduce the total completion time and execution time and increase the gain of providers along with the satisfaction of users by assigning priorities to the users who have paid the cost for the used resources to the service provider. One of the drawback of this work is that static threshold values are used. In [37], the authors have proposed a dynamic resource 
allocation framework for NFV-enabled MEC consisting of fast online heuristic-based incremental allocation mechanism and a re-optimization solution. The aim of this paper is to efficiently allocate all the resources among NFV-enabled MECs so that low latency and cost efficiency are achieved. Although, $33 \%$ of the cost can be saved by the proposed work as compared to existing solutions with xed-location MEC however, this framework does not assist multiple services of different performance requirements. In [38], a mathematical model of cloud computing is proposed by Ibrahim et al., in the economic fractional dynamic system. In the proposed model, agent will separately optimize the entire cost involving two mechanisms; the implementation cost of cloud computing pattern and the effort cost of shifting to the cloud computing pattern. However, hope bifurcation of economic systems is dependent on several other rules. In [39], the authors have proposed a cloud computing simulation model of the smart grid for hosting smart grid applications, determining VM/ cloudlet parameters, along with cost prediction and processing time for the smart grid tasks. However, security policies for smart grid cloud are ignored.

It can be seen clearly from this state-of-the-art work that there exist a lot of gaps which needs to be filled. In this regard, this work is based on minimizing cost while utilizing maximum amount of resources.

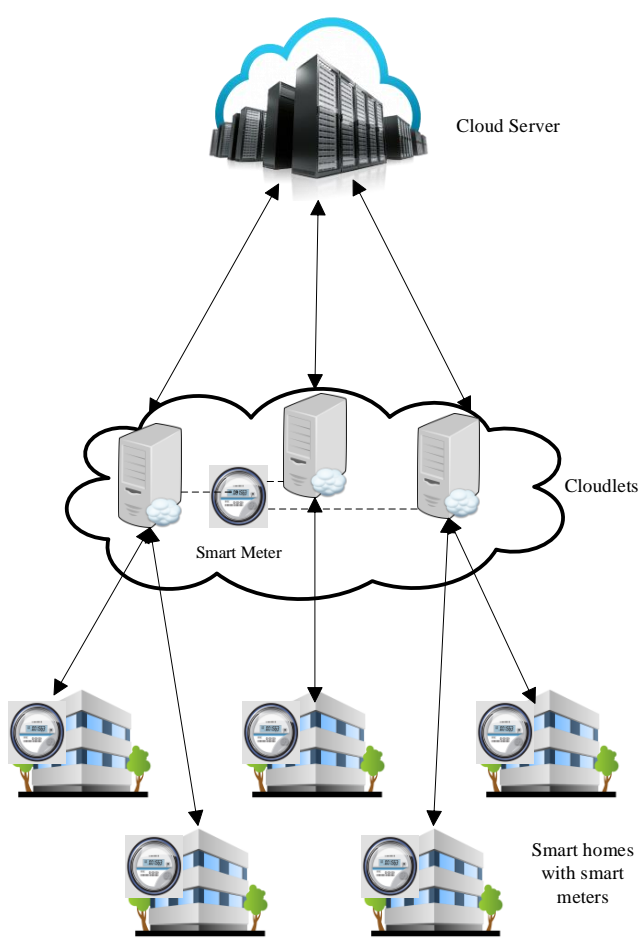

Fig. 2. Proposed cloudlet architecture.

\section{PROPOSED METHOdOLOGY}

In the proposed system, we apply PIO and BBA along with the ToU pricing scheme to estimate the bill of the cloudlet utilization. The task is accomplished by determining the energy

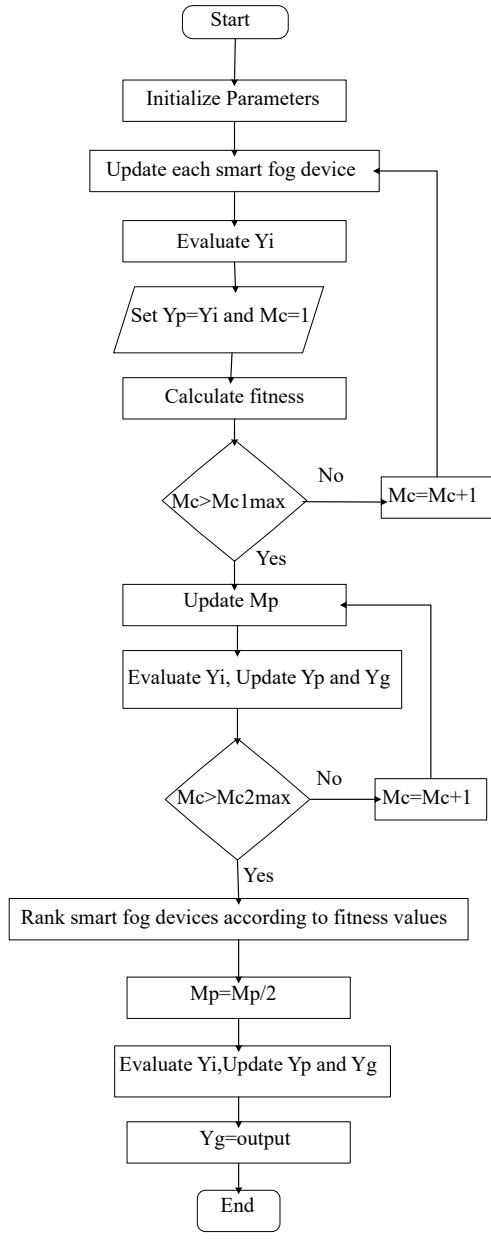

Fig. 3. Flowchart of PIO in the context of cloudlets.

consumption. Fig. 2 shows the proposed architecture of the cloudlets. The cloudlets and smart homes are connected with the smart meter to determine the energy consumed which is further used for bill estimation. The information about the amount of energy consumed by the smart homes is gathered through the smart meter and transmitted to the cloudlets. Eventually, the optimization technique is applied and optimal schedule of power consumption of all of the smart homes and cloudlets is examined. The cost is determined by estimating the amount of resources utilized by the clients by using pay as you go pricing model. The consumers can control the cost and energy consumed by optimal scheduling. For this, two bio-inspired optimization techniques, namely, PIO and BBA are used.

\section{A. Working of Optimization Techniques}

The working of the two bio-inspired techniques used for determining the bills is given in this section. The description of which is also given in the form of flowcharts.

1) Pigeon Inspired Optimization (PIO): Considering the homing behaviour of pigeons, Duan and Qiao proposed PIO in 2014 [9]. The PIO has a better optimization performance and a high convergence speed [40]. Two major operators used in this technique are: (i) Map and Compass Operator; and (ii) Landmark Operator. 
TABLE I. STATE-OF-THE-ART WORK

\begin{tabular}{|c|c|c|c|c|c|c|c|c|c|c|c|c|c|c|c|c|}
\hline \multicolumn{5}{|c|}{ Features } & \multicolumn{6}{|c|}{ Limitations } & \multicolumn{6}{|c|}{ Parameters } \\
\hline 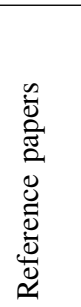 & $\overrightarrow{\tilde{\theta}}$ & 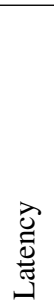 & 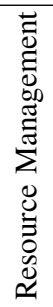 & 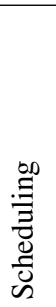 & 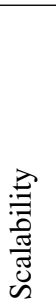 & 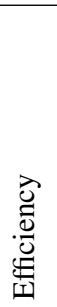 & 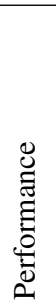 & ¿ & $\begin{array}{l}\vec{b} \\
0 \\
\vec{b} \\
\overrightarrow{0}\end{array}$ & 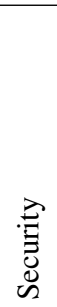 & 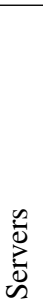 & 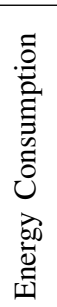 & 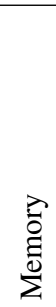 & 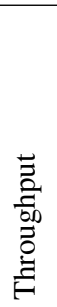 & $\underset{g}{\varrho}$ & 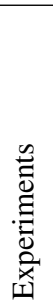 \\
\hline [10] & $\checkmark$ & $x$ & $x$ & $\sqrt{ }$ & $\sqrt{ }$ & $x$ & $x$ & $x$ & $x$ & $x$ & $\sqrt{ }$ & $x$ & $\sqrt{ }$ & $x$ & $\sqrt{ }$ & $\checkmark$ \\
\hline [11] & $\sqrt{ }$ & $\sqrt{ }$ & $\sqrt{ }$ & $x$ & $x$ & $x$ & $x$ & $x$ & $x$ & $\checkmark$ & $\checkmark$ & $x$ & $x$ & $\sqrt{ }$ & $x$ & $\checkmark$ \\
\hline [12] & $\checkmark$ & $x$ & $x$ & $\checkmark$ & $x$ & $\checkmark$ & $x$ & $x$ & $\checkmark$ & $x$ & $x$ & $x$ & $x$ & $\checkmark$ & $x$ & $\checkmark$ \\
\hline [13] & $\checkmark$ & $x$ & $x$ & $\checkmark$ & $\checkmark$ & $x$ & $x$ & $\checkmark$ & $\checkmark$ & $x$ & $x$ & $x$ & $x$ & $x$ & $\checkmark$ & $\checkmark$ \\
\hline [14] & $x$ & $x$ & $\checkmark$ & $x$ & $\sqrt{ }$ & $x$ & $x$ & $x$ & $x$ & $x$ & $\checkmark$ & $\checkmark$ & $x$ & $x$ & $x$ & $\checkmark$ \\
\hline [15] & $\checkmark$ & $x$ & $x$ & $x$ & $x$ & $\checkmark$ & $\checkmark$ & $x$ & $x$ & $x$ & $x$ & $x$ & $x$ & $\checkmark$ & $\checkmark$ & $\checkmark$ \\
\hline [16] & $x$ & $x$ & $\checkmark$ & $x$ & $x$ & $x$ & $x$ & $\checkmark$ & $x$ & $x$ & $\checkmark$ & $x$ & $x$ & $x$ & $\checkmark$ & $\checkmark$ \\
\hline [17] & $\sqrt{ }$ & $x$ & $\checkmark$ & $\sqrt{ }$ & $x$ & $\sqrt{ }$ & $\checkmark$ & $x$ & $\checkmark$ & $x$ & $\checkmark$ & $x$ & $x$ & $x$ & $x$ & $x$ \\
\hline [18] & $\checkmark$ & $\checkmark$ & $x$ & $x$ & $x$ & $x$ & $x$ & $\checkmark$ & $x$ & $x$ & $\checkmark$ & $\checkmark$ & $x$ & $\checkmark$ & $\checkmark$ & $\checkmark$ \\
\hline [19] & $\checkmark$ & $\checkmark$ & $x$ & $x$ & $x$ & $x$ & $x$ & $\checkmark$ & $x$ & $x$ & $\checkmark$ & $x$ & $\sqrt{ }$ & $x$ & $\checkmark$ & $x$ \\
\hline [20] & $\checkmark$ & $x$ & $\sqrt{ }$ & $\sqrt{ }$ & $x$ & $\checkmark$ & $\checkmark$ & $\checkmark$ & $x$ & $x$ & $x$ & $\checkmark$ & $x$ & $x$ & $\checkmark$ & $\checkmark$ \\
\hline [21] & $\checkmark$ & $x$ & $x$ & $x$ & $\checkmark$ & $x$ & $x$ & $x$ & $x$ & $x$ & $x$ & $x$ & $\checkmark$ & $\checkmark$ & $\checkmark$ & $\checkmark$ \\
\hline [22] & $\checkmark$ & $x$ & $x$ & $x$ & $x$ & $\checkmark$ & $\checkmark$ & $x$ & $x$ & $x$ & $x$ & $x$ & $x$ & $\checkmark$ & $\checkmark$ & $\checkmark$ \\
\hline [23] & $\checkmark$ & $x$ & $x$ & $\checkmark$ & $x$ & $x$ & $\checkmark$ & $x$ & $x$ & $x$ & $\bar{x}$ & $x$ & $x$ & $x$ & $\checkmark$ & $x$ \\
\hline [24] & $\checkmark$ & $x$ & $x$ & $\checkmark$ & $x$ & $x$ & $x$ & $\checkmark$ & $x$ & $x$ & $x$ & $x$ & $x$ & $x$ & $\checkmark$ & $x$ \\
\hline$[25]$ & $\checkmark$ & $x$ & $\checkmark$ & $x$ & $x$ & $x$ & $x$ & $x$ & $\checkmark$ & $x$ & $x$ & $x$ & $\checkmark$ & $x$ & $\checkmark$ & $\checkmark$ \\
\hline [26] & $\checkmark$ & $x$ & $\checkmark$ & $x$ & $x$ & $\checkmark$ & $\checkmark$ & $\checkmark$ & $\checkmark$ & $x$ & $x$ & $\sqrt{ }$ & $\checkmark$ & $x$ & $\checkmark$ & $\checkmark$ \\
\hline [27] & $\checkmark$ & $x$ & $\checkmark$ & $x$ & $\checkmark$ & $\checkmark$ & $x$ & $x$ & $\checkmark$ & $x$ & $x$ & $x$ & $\checkmark$ & $x$ & $x$ & $\checkmark$ \\
\hline$[28]$ & $x$ & & $\checkmark$ & $x$ & $\sqrt{ }$ & $\checkmark$ & $\checkmark$ & $x$ & $x$ & $x$ & $x$ & $\checkmark$ & $\checkmark$ & $x$ & $x$ & $x$ \\
\hline [29] & $\checkmark$ & $x$ & $\checkmark$ & $x$ & $x$ & $x$ & $x$ & $\checkmark$ & $x$ & $x$ & $x$ & $x$ & $x$ & $\checkmark$ & $\checkmark$ & $x$ \\
\hline [30] & $\checkmark$ & $x$ & $\checkmark$ & $x$ & $x$ & $\checkmark$ & $\checkmark$ & $x$ & $x$ & $x$ & $\checkmark$ & $x$ & $\checkmark$ & $x$ & $x$ & $\checkmark$ \\
\hline [31] & $\checkmark$ & $x$ & $x$ & $\checkmark$ & $x$ & $\checkmark$ & $x$ & $x$ & $\checkmark$ & $x$ & $x$ & $x$ & $x$ & $x$ & $\checkmark$ & $x$ \\
\hline [32] & $\checkmark$ & $x$ & $\checkmark$ & $x$ & $\checkmark$ & $x$ & $x$ & $x$ & $x$ & $x$ & $x$ & $x$ & $x$ & $\checkmark$ & $\checkmark$ & $\checkmark$ \\
\hline [33] & $\checkmark$ & $\checkmark$ & $x$ & $\checkmark$ & $\checkmark$ & $x$ & $x$ & $x$ & $x$ & $x$ & $x$ & $\checkmark$ & $x$ & $\checkmark$ & $x$ & $x$ \\
\hline [34] & $\checkmark$ & $x$ & $x$ & $\checkmark$ & $x$ & $x$ & $x$ & $x$ & $x$ & $\checkmark$ & $x$ & $\checkmark$ & $x$ & $x$ & $x$ & $x$ \\
\hline [35] & $\checkmark$ & $x$ & $\sqrt{ }$ & $\checkmark$ & $x$ & $x$ & $x$ & $\checkmark$ & $x$ & $x$ & $\sqrt{ }$ & $x$ & $x$ & $x$ & $\sqrt{ }$ & $\checkmark$ \\
\hline [36] & $\checkmark$ & $x$ & $\checkmark$ & $x$ & $\checkmark$ & $\checkmark$ & $x$ & $x$ & $x$ & $x$ & $\checkmark$ & $x$ & $x$ & $x$ & $\checkmark$ & $\checkmark$ \\
\hline [37] & $x$ & $x$ & $x$ & $x$ & $x$ & $x$ & $\checkmark$ & $x$ & $x$ & $x$ & $\checkmark$ & $x$ & $x$ & $x$ & $\checkmark$ & $\checkmark$ \\
\hline [38] & $\checkmark$ & $x$ & $x$ & $x$ & $x$ & $\sqrt{ }$ & $\sqrt{ }$ & $\checkmark$ & $\checkmark$ & $x$ & $x$ & $x$ & $x$ & $x$ & $x$ & $x$ \\
\hline [39] & $\checkmark$ & $\checkmark$ & $x$ & $x$ & $x$ & $x$ & $x$ & $x$ & $x$ & $\checkmark$ & $\checkmark$ & $x$ & $x$ & $x$ & $\checkmark$ & $\checkmark$ \\
\hline
\end{tabular}

\section{i) Map and Compass Operator}

Parameter initialization along with random generation of population is done. Then fitness is calculated. Pigeons use magneto-reception to judge the earths magnetic field. They depend on sun and magnetic particles while moving towards destination.

\section{ii) Landmark Operator}

The pigeons fly near to their target by leaning on the adjacent landmarks. If the landmarks are known to them, they will fly to their goal directly. On contrary, if the landmarks are unknown to them and pigeons are far apart from their destination then they will track the pigeons who are familiar with the landmarks. Using this operator, half of the population is stranded after sorting according to the fitness function. This is how pigeons find food and in this case, we will find an optimal solution i.e., the cost estimation. Fig. 3 shows the working of the PIO technique. Some of the steps are taken from [9] in order to find the global best solution using a fitness function.

2) Binary Bat Algorithm (BBA): Bat Algorithm (BA) was proposed by Xin-She Yang in 2010 [4] for solving the complex optimization problems. Inspired from the echolocative behavior of bats global optimization is performed. Binary version of BA i.e., BBA [5] uses artificial bats navigating and hunting in binary search spaces by switching their positions between 0 to 1 values. Bats use natural sonar in order to navigate and hunt. When finding the prey bats adopt two main characteristics. While chasing the prey bats tend to decrease the loudness and increase the emission rate of ultrasonic sound. It has been proven that BBA is capable of providing competitive results as compared to most well known algorithms such as Particle Swarm Optimization (PSO) and Genetic Algorithm (GA) in terms of convergence speed and improved local optima avoidance [5], [6]. As stated by Mirjalili et al., BBA has fastest convergence rate along with high performance in finding global solutions. 


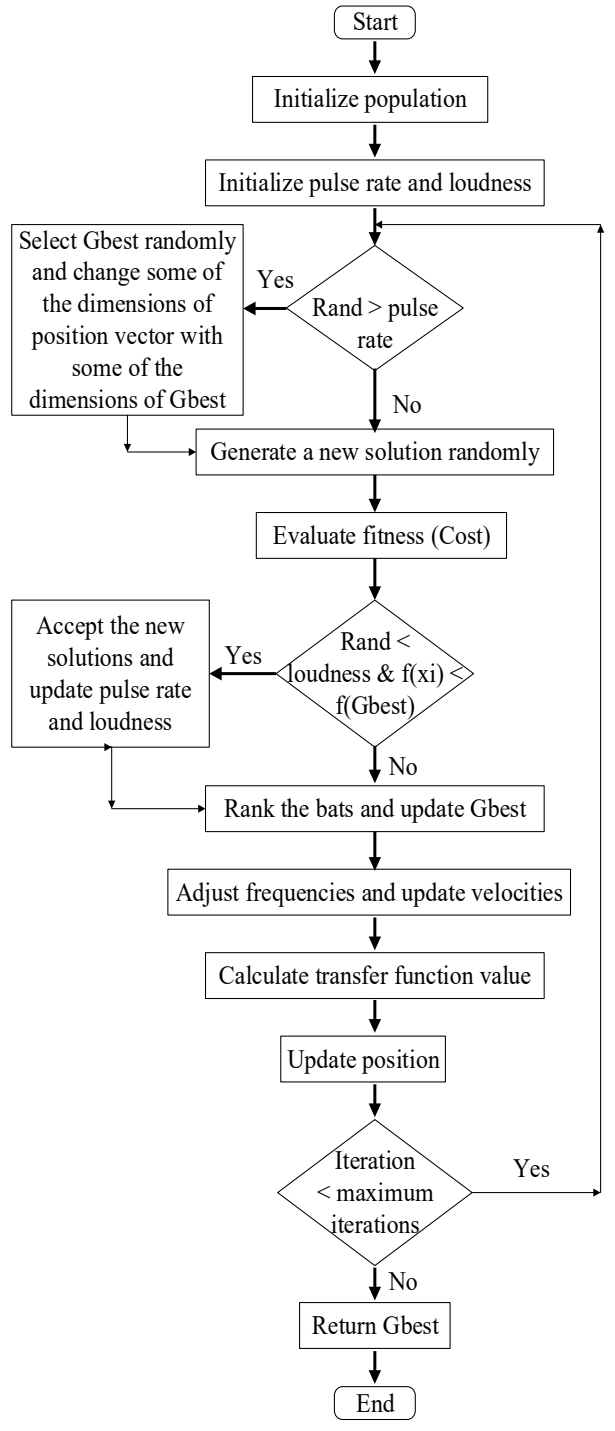

Fig. 4. Flowchart of BBA in the context of cloudlets.

The working of the BBA is given in Fig. 4, where fitness is considered as the cost and the Gbest is the one with the minimum cost.

\section{Simulations AND Results}

In order to evaluate the bio-inspired techniques for the purpose of estimation of bills, MATLAB is used. Simulations are held in MATLAB for ease of visualization by setting up a Fog computing environment, leveraging six fog nodes. Simulations are held by considering varying values of power consumption along with cloudlets operational time in which resources are being utilized. Experiments are conducted under similar conditions, i.e., under equal amount of load both PIO and BBA are compared and evaluated.

Along with the PIO and the BBA, ToU pricing signal is used to lessen the bill of the cloudlets. The scheduling of the cloudlets is done in order to utilize resources efficiently so that overall cost can be reduced.

Fig. 5, shows the resources utilized by users. With the increased resource utilization, cost will also be increased.

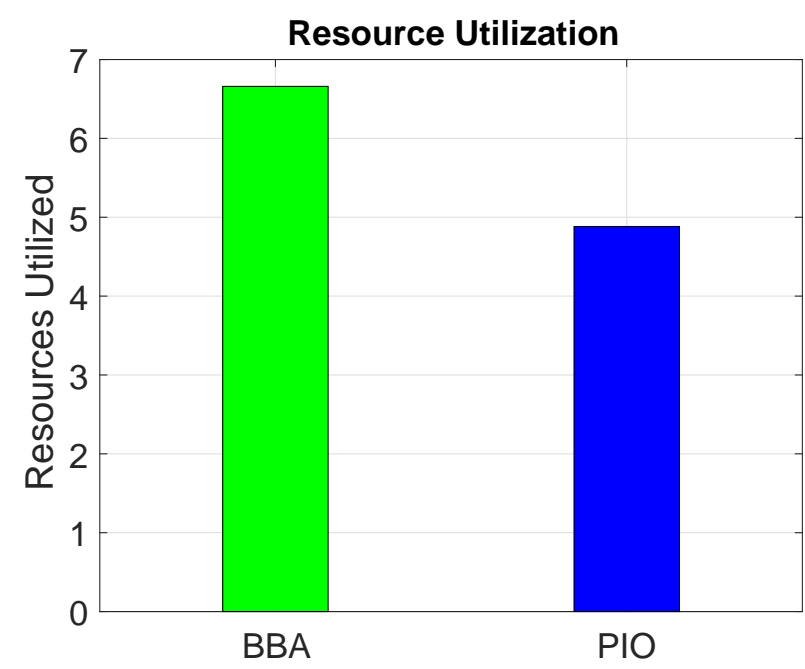

Fig. 5. Comparison of resource utilization for PIO and BBA.

Results clearly show that by using the BBA algorithm, more resources can be acquired as compared to the PIO. Around $25 \%$ more resources can be used in BBA in contrast to PIO.

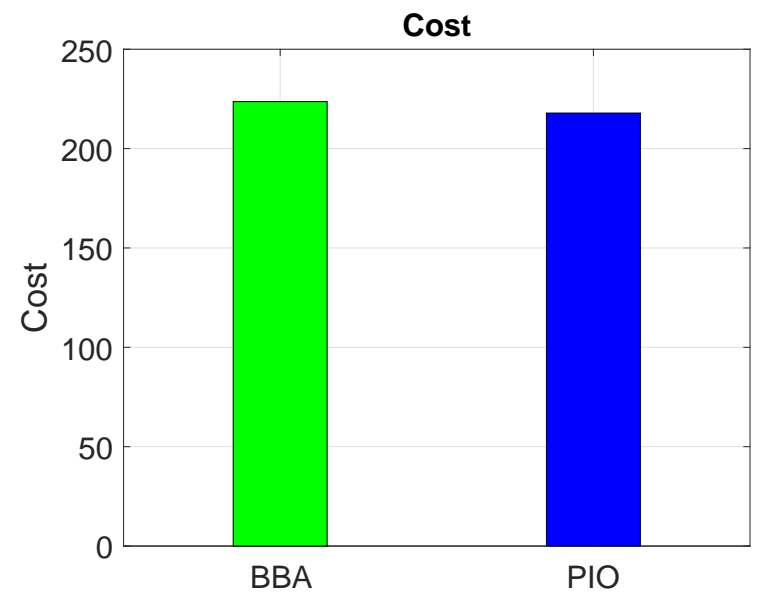

Fig. 6. Comparison of utilization cost for PIO and BBA.

In Fig. 6, cost of the resources which are utilized is given. This shows that the cost of BBA is little bit more than that of PIO. This is because through BBA more resources can be utilized in comparison with PIO. Therefore, consumers have to pay for the utilized resources. Although BBA requires a bit more cost as compared to PIO, still BBA is better as a lot more resources can be acquired using $\mathrm{BBA}$ with a minor increase in cost.

Fig. 7, represents the hourly cost of the resources utilized by the consumers. From figure, it can be seen that highest peaks are formed by the PIO throughout. At a certain point of the day BBA may result in high cost however, throughout the day cost of PIO is more i.e., highest peaks are formed by PIO. The maximum cost is increased till 54 cents while using BBA the cost is less throughout the day in contrast to PIO. 


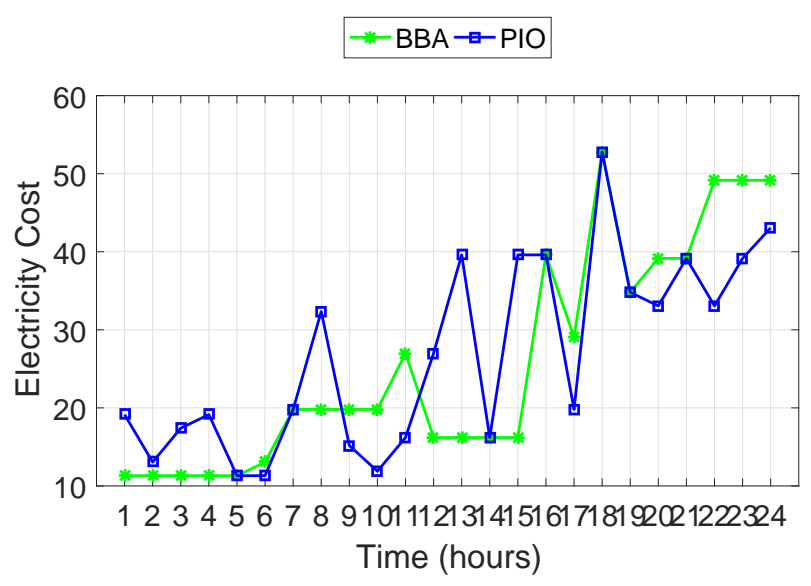

Fig. 7. Hourly cost for the simulation.

\section{CONCLUSIONS}

In this paper, two nature inspired algorithms were compared and evaluated regarding their performance in means of estimation of utility bills based upon the usage of cloudlets. The Binary Bat Algorithm (BBA) and Pigeon Inspired Optimization (PIO) along with Time of Use pricing signal were used for cost estimation. The evaluation is done while considering the resources utilized by the consumers and thus their hourly consumption cost. Simulation results show that the BBA can efficiently utilize large amount of resources i.e., $25 \%$ as compared to PIO, therefore, it has a minor increase in cost as compared to the PIO. The cost of BBA is approximately $5 \%$ more than that of the PIO. From the simulation results, we conclude that the overall performance of BBA is better than PIO, as with only $5 \%$ increase in cost $25 \%$ more resources can be utilized. These techniques lead to cost estimation based upon the resources utilized by the cloudlets. In future, we plan to perform experiments using other noteworthy bio-inspired optimization techniques, such as Earth Worm Optimization Algorithm (EWA), Bellman-Ford, Beetle Antennae Search Algorithm (BAS) for utility pricing where the simulations would be setup in a dominated environment fixed in devoted simulator such as iFogsim.

\section{REFERENCES}

[1] Bonomi, Flavio, R. Milito, J. Zhu, and S. Addepalli. "Fog computing and its role in the internet of things." In Proceedings of the first edition of the MCC workshop on Mobile cloud computing, pp. 13-16. ACM, 2012.

[2] Rottondi, Cristina, A. Barbato, L. Chen, and G. Verticale. "Enabling privacy in a distributed game-theoretical scheduling system for domestic appliances." IEEE Transactions on Smart Grid 8, no. 3 (2017): 12201230 .

[3] Alam, M. Raisul, M. B. Ibne Reaz, and M. A. Mohd Ali. "A review of smart homesPast, present, and future." IEEE Transactions on Systems, Man, and Cybernetics, Part C (Applications and Reviews) 42, no. 6 (2012): 1190-1203.

[4] Yang, Xin-She. "A new metaheuristic bat-inspired algorithm." In Nature inspired cooperative strategies for optimization (NICSO 2010), pp. 6574. Springer, Berlin, Heidelberg, 2010.

[5] Mirjalili, Seyedali, S. M. Mirjalili, and X. S. Yang. "Binary bat algorithm." Neural Computing and Applications 25, no. 3-4 (2014): 663-681.
[6] Yang, Xin-She, and X. He. "Bat algorithm: literature review and applications." International Journal of Bio-Inspired Computation 5, no. 3 (2013): 141-149.

[7] Arshad, Hafsa, S. Batool, Z. Amjad, M. Ali, S. Aimal, and N. Javaid. "Pigeon Inspired Optimization and Enhanced Differential Evolution Using Time of Use Tariff in Smart Grid." In International Conference on Intelligent Networking and Collaborative Systems, pp. 563-575. Springer, Cham, 2017.

[8] Amjad, Zunaira, S. Batool, H. Arshad, K. Parvez, M. Farooqi, and N. Javaid. "Pigeon Inspired Optimization and Enhanced Differential Evolution in Smart Grid Using Critical Peak Pricing." In International Conference on Intelligent Networking and Collaborative Systems, pp. 505-514. Springer, Cham, 2017.

[9] Duan, Haibin, and P. Qiao. "Pigeon-inspired optimization: a new swarm intelligence optimizer for air robot path planning." International Journal of Intelligent Computing and Cybernetics 7, no. 1 (2014): 24-37.

[10] Pham, X. Qui, N. D. Man, N. D. T. Tri, N. Q. Thai, and E. N. Huh. ”A cost-and performance-effective approach for task scheduling based on collaboration between cloud and fog computing." International Journal of Distributed Sensor Networks 13, no. 11 (2017): 1550147717742073.

[11] Yang, Lei, J. Cao, G. Liang, and X. Han. "Cost aware service placement and load dispatching in mobile cloud systems." IEEE Transactions on Computers 65, no. 5 (2016): 1440-1452.

[12] Sidhu, H. Singh. "Cost-deadline based task scheduling in cloud computing." In Advances in Computing and Communication Engineering (ICACCE), 2015 Second International Conference on, pp. 273-279. IEEE, 2015.

[13] Kang, D. Ki, S. H. Kim, C. H. Youn, and M. Chen. "Cost adaptive workflow scheduling in cloud computing." In Proceedings of the 8th International conference on ubiquitous information management and communication, p. 65. ACM, 2014.

[14] Aadkane, Trapti, and S. Monga. "An Energy Efficient Cost Aware Virtual Machine Migration Approach for the Cloud Environment."

[15] Aslanpour, M. Sadegh, M. G. Arani, and A. N. Toosi. "Auto-scaling web applications in clouds: a cost-aware approach.” Journal of Network and Computer Applications 95 (2017): 26-41.

[16] Aazam, Mohammad, and E. N. Huh. "Broker as a service (baas) pricing and resource estimation model." In Cloud Computing Technology and Science (CloudCom), 2014 IEEE 6th International Conference on, pp. 463-468. IEEE, 2014

[17] Kim, S. Hwan, D. K. Kang, W. J. Kim, M. Chen, and C. H. Youn. "A science gateway cloud with cost-adaptive VM management for computational science and applications." IEEE Systems Journal 11, no. 1 (2017): 173-185.

[18] Kim, Yeongiin, J. Kwak, and S. Chong. "Dual-Side Optimization for Cost-Delay Tradeoff in Mobile Edge Computing." IEEE Transactions on Vehicular Technology 67, no. 2 (2018): 1765-1781.

[19] Kim, Yeongjin, J. Kwak, and S. Chong. "Dual-side dynamic controls for cost minimization in mobile cloud computing systems." In Modeling and Optimization in Mobile, Ad Hoc, and Wireless Networks (WiOpt), 2015 13th International Symposium on, pp. 443-450. IEEE, 2015.

[20] Cheng, Mingxi, J. Li, and S. Nazarian. "DRL-cloud: Deep reinforcement learning-based resource provisioning and task scheduling for cloud service providers." In Design Automation Conference (ASP-DAC), 2018 23rd Asia and South Pacific, pp. 129-134. IEEE, 2018.

[21] Kansal, Sahil, H. Kumar, S. Kaushal, and A. K. Sangaiah. "Genetic algorithm-based cost minimization pricing model for on-demand IaaS cloud service." The Journal of Supercomputing (2018): 1-26.

[22] Shahapure, Nagamani H., and P. Jayarekha. "Load balancing with optimal cost scheduling algorithm." In Computation of Power, Energy, Information and Communication (ICCPEIC), 2014 International Conference on, pp. 24-31. IEEE, 2014.

[23] Li, Ji, Y. Wang, T. Cui, S. Nazarian, and M. Pedram. "Negotiationbased task scheduling to minimize users electricity bills under dynamic energy prices." In Green Communications (OnlineGreencomm), 2014 IEEE Online Conference on, pp. 1-6. IEEE, 2014.

[24] Li, Ji, Y. Wang, X. Lin, S. Nazarian, and M. Pedram. "Negotiationbased task scheduling and storage control algorithm to minimize user's electric bills under dynamic prices." In Design Automation Conference (ASP-DAC), 2015 20th Asia and South Pacific, pp. 261-266. IEEE, 2015. 
[25] Patel, Suhradam, R. K. Bhujade, A. Sinhal, and S. Kathrotia. "Resource optimization and cost reduction by dynamic virtual machine provisioning in cloud." In Advances in Computing, Communications and Informatics (ICACCI), 2013 International Conference on, pp. 857-861. IEEE, 2013.

[26] Xia, Qiufen, W. Liang, and W. Xu. "Throughput maximization for online request admissions in mobile cloudlets." In Local Computer Networks (LCN), 2013 IEEE 38th Conference on, pp. 589-596. IEEE, 2013.

[27] Sturm, Tobias, F. Jrad, and A. Streit. "Storage CloudSim." In Proceedings of the 4th International Conference on Cloud Computing and Services Science, pp. 186-192. SCITEPRESS-Science and Technology Publications, Lda, 2014.

[28] Youn, C. Hyun, M. Chen, and P. Dazzi. "VM Placement via Resource Brokers in a Cloud Datacenter." In Cloud Broker and Cloudlet for Workflow Scheduling, pp. 47-73. Springer, Singapore, 2017.

[29] Cao, Zijian, J. Lin, C. Wan, Y. Song, Y. Zhang, and X. Wang. "Optimal cloud computing resource allocation for demand side management in smart grid." IEEE Transactions on Smart Grid 8, no. 4 (2017): 19431955.

[30] Singh, Sukhpal, and I. Chana. "Q-aware: Quality of service-based cloud resource provisioning." Computers and Electrical Engineering 47 (2015): $138-160$

[31] Zhao, Jian, H. Li, C. Wu, Z. Li, Z. Zhang, and F. CM Lau. "Dynamic pricing and profit maximization for the cloud with geo-distributed data centers." In INFOCOM, 2014 Proceedings IEEE, pp. 118-126. IEEE, 2014.

[32] Sheeja, Y. S., and S. Jayalekshmi. "Cost effective load balancing based on honey bee behaviour in cloud environment." In Computational Systems and Communications (ICCSC), 2014 First International Conference on, pp. 214-219. IEEE, 2014.
[33] Ren, Shaolei, and M. V. D. Schaar. "Dynamic scheduling and pricing in wireless cloud computing." IEEE Transactions on Mobile Computing 13, no. 10 (2014): 2283-2292.

[34] Yaghmaee, M. Hossein, M. Moghaddassian, and A. L. Garcia. "Power Consumption Scheduling for Future Connected Smart Homes Using BiLevel Cost-Wise Optimization Approach.” In Smart City 360, pp. 326338. Springer, Cham, 2016.

[35] Chopra, Nitish, and S. Singh. "Deadline and cost based workflow scheduling in hybrid cloud." In Advances in Computing, Communications and Informatics (ICACCI), 2013 International Conference on, pp. 840-846. IEEE, 2013.

[36] Pandey, Manish, and S. K. Verma. "Cost based resource allocation strategy for the cloud computing environment." In Computing, Communication and Networking Technologies (ICCCNT), 2017 8th International Conference on, pp. 1-7. IEEE, 2017.

[37] Yang, Binxu, W. K. Chai, Z. Xu, K. V. Katsaros, and G. Pavlou. "CostEfficient NFV-Enabled Mobile Edge-Cloud for Low Latency Mobile Applications." IEEE Transactions on Network and Service Management (2018).

[38] Ibrahim, Rabha W., and A. Gani. "A mathematical model of cloud computing in the economic fractional dynamic system." Iranian Journal of Science and Technology, Transactions A: Science 42, no. 1 (2018): 65-72.

[39] Mehmi, Sandeep, H. K. Verma, and A. L. Sangal. "Simulation modeling of cloud computing for smart grid using CloudSim." Journal of Electrical Systems and Information Technology 4, no. 1 (2017): 159-172.

[40] Khan, Nasir, N. Javaid, M. Khan, A. Subhani, A. Mateen, and A. Iqbal. "Harmony Pigeon Inspired Optimization for Appliance Scheduling in Smart Grid." 